\title{
Quadratic Programming Vs. Concurrent Correlation Matching under Non-Uniform Image Contrast and Offset
}

\author{
G. Gimel'farb, P. Delmas, A. Shorin, J. Morris \\ Computer Science, University of Auckland, New Zealand. \\ Email: \{ggim001, pdel016, al, jmor159\}@cs .auckland.ac.nz
}

\begin{abstract}
Recognition of objects-of-interest by similarity of prototypes (templates) to sensed images of natural scenes is important in computer vision, digital photogrammetry and multimedia data retrieval. However, accurate template matching in the presence of non-uniform nuisance contrast and offset deviations remains a challenging problem. Uniform deviations leading to cross-correlation matching scores have been studied for decades, but non-uniform ones have been little explored - mostly, only computationally intensive gradientbased matching for low order polynomial deviation model. This paper presents two computationally simpler alternative approaches: (i) an analytical least-squares template matching for polynomial deviation models of an arbitrary order using a bank of concurrent cross-correlation scores and (ii) a fast numerical quadratic programming based matching for a new general model of loosely constrained non-uniform contrast and offset deviations. Experiments confirm that these approaches hold great promise in practice.
\end{abstract}

Keywords: Template matching, polynomial contrast, non-uniform contrast, concurrent correlation, quadratic programming

\section{Introduction}

Robust template matching is a basic part of any image processing, computer vision, photogrammetric and multimedia system that has to detect, classify or retrieve objects on the basis of sensed 1D signals or 2D images of natural scenes. Typical matching frameworks assume that the sensed data mostly follow probability models that account for varying environments and sensing or imaging conditions. The most popular cross-correlation matching assumes uniform contrast deviations of a template in the presence of an additive, independent, central-symmetric and random noise $[1,2]$. However, in practice, these assumptions frequently do not hold and non-uniform contrast deviations that exist even in a controlled real world environment (e.g. due to changing illumination) often make the correlation rather useless.

A polynomial model of non-uniform contrast introduced by Lai [3] stimulated a number of subsequent efforts $[4,5,6]$. Instead of the more conventional but not robust least squares estimator, Lai's matching uses the robust M-estimator [7] and thus a computationally complex numerical gradient search for the best match in the parameter space of both geometric transformation and polynomial coefficients. This high dimensional space is

978-1-4244-2582-2/08/\$25.00 (C)2008 IEEE difficult to explore extensively, so various simplifications were made such as a heuristic feature sampling with manual changes of selection parameters for different recognition tasks [6] or matching only contour images after edge detection [5]. Thus the approach was practical with only a small number of polynomial coefficients and failed with non-smooth contrast changes and even shadows [3]. Low order polynomial contrast deviations tend to have a rather unnatural visual appearance (Fig. 1(c)): even small movements of light sources lead to large jumps in intensity (due to shadows and reflections) which cannot be adequately modelled with simple polynomial functions. Fig. 2(c) shows contrast deviations for a small $\left(15^{\circ}\right)$ rotation of the light source.

We show that the least-squares framework allows us to reduce matching based on an arbitrary order

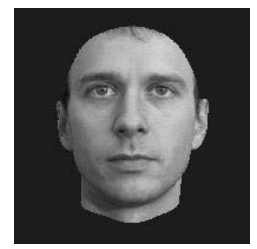

(a) Template

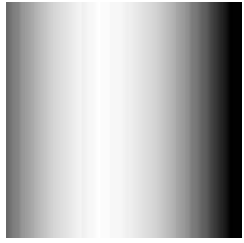

(b) Polynomial

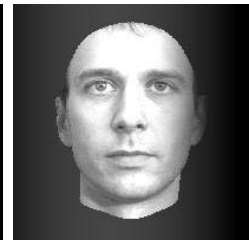

(c) Deviation
Figure 1: (a) Template from the MIT database [8], (b) (synthetic) example of scaled parabolic contrast factors, and (c) contrast deviations using this model. 

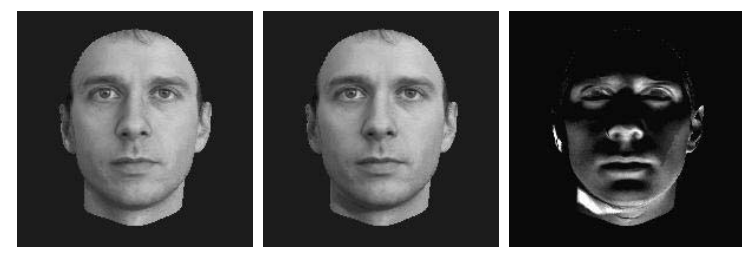

Figure 2: Contrast variations with small change in source position: (a) frontal illumination, (b) illumination from $15^{\circ}$, (c) scaled contrast deviations between (a) and (b).

polynomial contrast and offset model to a simple concurrent cross-correlation matching with a bank of specific co-registered templates. This reduction simplifies the elimination of nuisance deviations from a similarity score between an image and its template. Also, a new general model of loosely constrained non-uniform contrast and offset deviations from a template that preserve its visual appearance as well as a computationally feasible numerical least-squares matching framework based on quadratic programming (QP) are proposed. Both QP-matching and concurrent correlation matching can be made robust in the presence of outliers that violate the underlying probability model of central-symmetric noise, much as the conventional least-squares correlation in previous work $[9,10]$.

The paper is organised as follows: concurrent correlation matching for polynomial deviation models is described in Section 2. Section 3 introduces a new model of general constrained non-uniform contrast and offset deviations that more closely fits natural images than polynomial models. This model leads to QP-based matching and a fast numerical implementation. Experimental results and conclusions are presented in Section 4.

\section{Concurrent Correlation under Polynomial Deviations}

Let $\mathcal{R}=[(x, y): x=0, \ldots, X-1 ; y=0, \ldots, Y-1]$ be a finite arithmetic lattice of $X Y$ pixels supporting images, and $\mathcal{Q}=\{0,1, \ldots, Q-1\}$ be a finite set of grey levels. Let $g: \mathcal{R} \rightarrow \mathcal{Q}$ denote a greyscale digital image, $t: \mathcal{R} \rightarrow \mathcal{Q}$, a template image to be matched to $g$, and $a: \mathcal{R} \rightarrow \mathcal{Q}$, an 'image' of admissible contrast and offset deviations from $t$. The images as vectors of the size $X Y$ are boldfaced $(\mathbf{g}, \mathbf{t}, \mathbf{a})$.

The $n^{\text {th }}$ order polynomial model of non-uniform contrast and offset deviations from $t$ is:

$$
a_{x, y}=\sum_{\substack{i, j=0 \\ i+j \leq n}}^{n}\left(\alpha_{i j} t_{x, y}+\beta_{i j}\right) x^{i} y^{j} \equiv \sum_{\kappa=1}^{k_{n}} \gamma_{\kappa} p_{x, y}^{[\kappa]}
$$
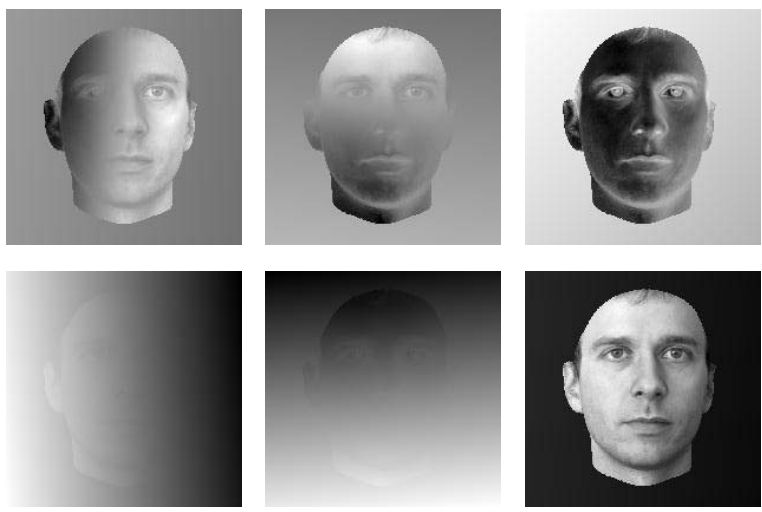

Figure 3: Bank of six eigen-templates for the $1^{\text {st }}$ order polynomial model of the template in Fig. 1(a)

specifies a vector space $\mathcal{A}(t ; E)$ of the admissible transformed templates a where $E$ denotes a $k_{n^{-}}$ dimensional space of the real-valued coefficients $\gamma_{\kappa}$ (i.e. $\alpha_{i j}$ and $\beta_{i j}$ ), e.g. $k_{1}=6$ for the first order model $(n=1)$ with the coefficients $\gamma_{1} \equiv \alpha_{00}, \gamma_{2} \equiv$ $\alpha_{10}, \ldots$, and $\gamma_{6} \equiv \beta_{01} ; k_{2}=12$, etc. Note that the offset term, $\beta$, is not considered by Lai[3]. Each image $\mathbf{a}$ in Eq. (1) is a linear combination of the $k_{n}$ component images: $\mathbf{p}^{[\kappa]}=\left[x^{i} y^{j} t_{x, y}:(x, y) \in \mathcal{R}\right]$ if $\gamma_{\kappa} \equiv \alpha_{i j}$ and $\mathbf{p}_{[\kappa]}=\left[x^{i} y^{j}:(x, y) \in \mathcal{R}\right]$ if $\gamma_{\kappa} \equiv \beta_{i j}$. Thus the image space $\mathcal{A}(t ; E)$ is a linear manifold of the maximal cardinality specified by the number of orthonormal vectors in it. As Fig. 1(c) suggests, a majority of the coefficient vectors $\gamma$ in $E$ produce unnatural distorted images $\mathbf{a} \in \mathcal{A}(t ; E)$.

With admissible deviations defined by Eq. (1), the least-squares matching of a sensed image $g$ to the template $t$ reduces to estimation of the $k_{n}$ minimisers, $\gamma_{\kappa}$, i.e. $\alpha_{i j}$ and $\beta_{i j}$, of the Cartesian distance between $g$ and $a \in \mathcal{A}(t ; E)$ :

$$
d(g, t \mid E)=\min _{a \in \mathcal{A}(t ; E)} \sum_{(x, y) \in \mathcal{R}}\left(a_{x, y}-g_{x, y}\right)^{2}
$$

This least-squares matching is equivalent to maximising the variance of the projection of $\mathbf{g}$ on the manifold $\mathcal{A}(t ; E)$. It is easily shown that the maximiser $\gamma$ is obtained by solving a system of linear equations $\gamma \mathbf{B}=\mathbf{b}$ with the $k_{n} \times k_{n}$ matrix $\mathbf{B}$ and the $k_{n}$-vector $\mathbf{b}$ :

$$
\begin{aligned}
\mathbf{B} & =\left[B_{\kappa l}=\sum_{(x, y) \in \mathcal{R}} p_{x, y}^{[\kappa]} p_{x, y}^{[l]}\right]_{\kappa, l=1}^{k_{n}} \\
\mathbf{b} & =\left[b_{\kappa}=\sum_{(x, y) \in \mathcal{R}} p_{x, y}^{[\kappa]} g_{x, y}: \kappa=1, \ldots, k_{n}\right]^{\top}
\end{aligned}
$$

The solution is simplified after replacing the $k_{n}$ component images $\mathbf{p}^{[\kappa]}$ with an orthogonal basis of the manifold $\mathcal{A}(t ; E)$ being built from the component images by using the tractable PCA [11]. The eigenvectors with non-zero eigenvalues (called the eigen-templates below) lead to a bank of $k_{n}$ orthonormal eigen-templates $\mathbf{e}_{l} ; l=1, \ldots, k_{n}$ for the concurrent cross-correlation matching. Then 

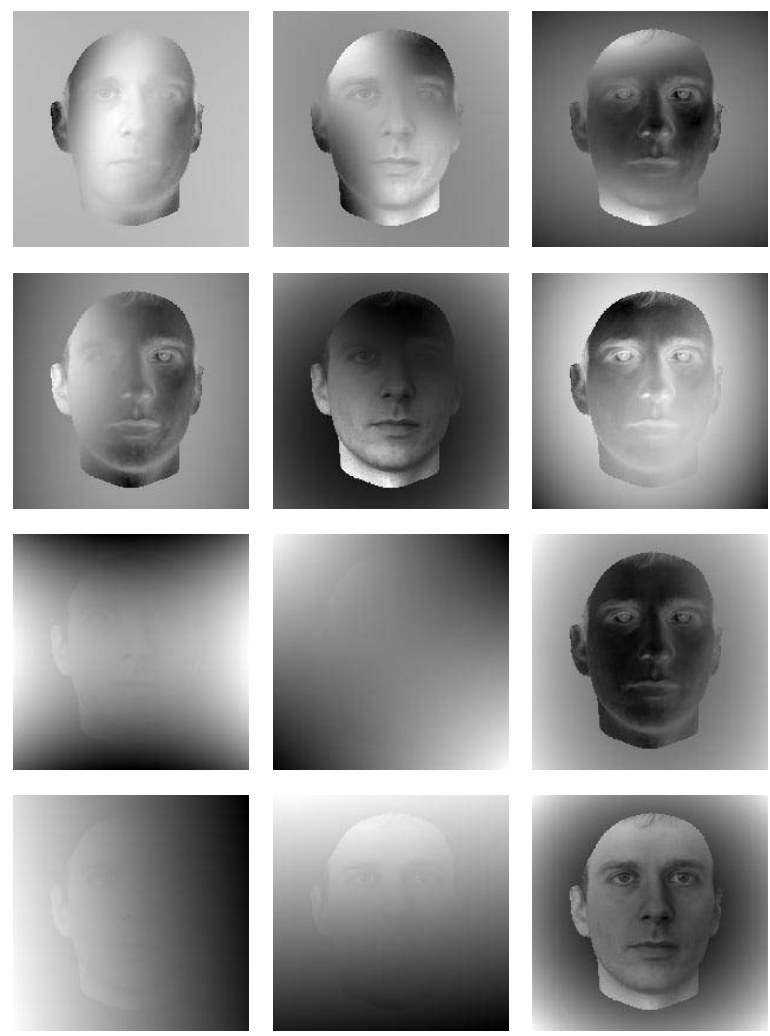

Figure 4: Bank of 12 eigen-templates for the $2^{\text {nd }}$ order polynomial model of the template in Fig. 1(a)

the minimisers are $\gamma_{\kappa}=\mathbf{e}_{\kappa}^{\top} \mathbf{g} ; \kappa=1, \ldots, k_{n}$ : the matching score is $d(g, t \mid E)=\mathbf{g}^{\top} \mathbf{g}-\sum_{\kappa=1}^{k_{n}} \gamma_{\kappa}^{2}$ and the closest to $\mathbf{g}$ transformed template $\mathbf{a}$ is $\mathbf{a}=$ $\sum_{\kappa=1}^{k_{n}} \gamma_{\kappa} \mathbf{e}_{\kappa}$. Figures 3 and 4 show the six and twelve grey-coded eigen-images for the first and second order polynomial deviation model, respectively, of the template $\mathbf{t}$ in Fig. 1(a). Note that the number of eigen-templates for the $i^{\text {th }}$ order model grows as $(i+1)(i+2)$, i.e. 20 and 30 eigen-templates for the $3^{\text {rd }}$ and $4^{\text {th }}$ order models, respectively.

\section{QP-Based Matching for Gen- eral Non-Uniform Deviations}

Limitations of low-order polynomial models can be overcome with a more general non-parametric model of spatially variant image contrast and offset. Let $\delta g_{\mathrm{h}: x, y}=g_{x ; y}-g_{x-1 ; y}$ and $\delta g_{\mathrm{v}: x, y}=$ $g_{x, y}-g_{x, y-1}$ denote grey level differences in the image $g$ between the nearest horizontal and vertical neighbours, respectively (with the like notation for $t$ and $a: \delta t_{\mathrm{h}: x, y}=t_{x ; y}-t_{x-1 ; y}, \delta a_{\mathrm{h}: x, y}=a_{x ; y}-$ $a_{x-1 ; y}, \delta t_{\mathrm{v}: x, y}=t_{x, y}-t_{x, y-1}$, and $\delta a_{\mathrm{v}: x, y}=a_{x, y}-$ $\left.a_{x, y-1}\right)$. The admissible contrast and offset deviations of $a$ from $t$ are specified with a fixed range $E=\left[e_{\min }, e_{\max }\right] ; 0<e_{\min } \leq e_{\max }$, of factors constraining the corresponding differences:

$$
\begin{aligned}
\Delta_{\mathrm{h}: x, y} & \leq \delta a_{\mathrm{h} x, y} \leq \bar{\Delta}_{\mathrm{h}: x, y} \\
\underline{\Delta}_{\mathrm{v}: x, y} & \leq \delta a_{\mathrm{v}: x, y} \leq \bar{\Delta}_{\mathrm{v}: x, y} \\
0 & \leq \quad h_{x ; y} \leq Q-1
\end{aligned}
$$

where

$$
\begin{aligned}
& \underline{\Delta}_{\mathrm{h}: x, y}= \begin{cases}e_{\min } \delta t_{\mathrm{h}: x, y} & \text { if } \delta t_{\mathrm{h}: x, y}>0 \\
e_{\max } \delta t_{\mathrm{h}: x, y} & \text { otherwise }\end{cases} \\
& \bar{\Delta}_{\mathrm{h}: x, y}= \begin{cases}e_{\max } \delta t_{\mathrm{h}: x, y} & \text { if } \delta t_{\mathrm{h}: x, y}>0 \\
e_{\min } \delta t_{\mathrm{h}: x, y} & \text { otherwise }\end{cases} \\
& \underline{\Delta}_{\mathrm{v}: x, y}= \begin{cases}e_{\min } \delta t_{\mathrm{v}: x, y} & \text { if } \delta t_{\mathrm{v}: x, y}>0 \\
e_{\max } \delta t_{\mathrm{v}: x, y} & \text { otherwise }\end{cases} \\
& \bar{\Delta}_{\mathrm{v}: x, y}= \begin{cases}e_{\max } \delta t_{\mathrm{v}: x, y} & \text { if } \delta t_{\mathrm{v}: x, y}>0 \\
e_{\min } \delta t_{\mathrm{v}: x, y} & \text { otherwise }\end{cases}
\end{aligned}
$$

Let $\mathcal{A}(t ; E)$ denote the set of all the images $a$ having the admissible deviations of Eq. (3) from the template $t$. For this general contrast and offset model, the least-squares matching of $g$ to $t$ reduces to the quadratic programming $(\mathrm{QP})$ minimisation of the Cartesian distance between $g$ and $a$ complying with the linear constraints of Eq. (3):

$$
d(g, t \mid E)=\min _{a \in \mathcal{A}(t ; E)} \sum_{(x, y) \in \mathcal{R}}\left(a_{x ; y}-g_{x ; y}\right)^{2}
$$

Due to large sparse matrices involved, the QP problem of Eqs. (5) and (3) may be solved conveniently with the Hildreth - D'Esopo algorithm [12, 13, 14] that reduces the original QP problem to a simple dual problem. Let us rewrite the original problem substituting $f_{x, y}=a_{x, y}-g_{x, y}$ and using the Lagrangian

$$
\sum_{(x, y) \in \mathcal{R}} f_{x ; y}^{2}+\sum_{i=1}^{6} u_{i: x, y} \psi_{i: x, y}
$$

where $u_{i: x, y} \geq 0 ; i=1, \ldots, 6$, are the nonnegative Lagrange multipliers for the constraints $\psi_{i: x, y} \leq 0$ :

$$
\begin{aligned}
& \psi_{1: x, y}=f_{x, y}-f_{x-1, y}-\bar{\Delta}_{\mathrm{h}: x, y}+\delta g_{\mathrm{h}: x, y} \\
& \psi_{2: x, y}=-f_{x, y}+f_{x-1, y}+\underline{\Delta}_{\mathrm{h}: x, y}-\delta g_{\mathrm{h}: x, y} \\
& \psi_{3: x, y}=f_{x, y}-f_{x, y-1}-\bar{\Delta}_{\mathrm{v}: x, y}+\delta g_{\mathrm{v}: x, y} \\
& \psi_{4: x, y}=-f_{x, y}+f_{x, y-1}+\underline{\Delta}_{\mathrm{v}: x, y}-\delta g_{\mathrm{v}: x ; y} \\
& \psi_{5: x, y}=f_{x, y}-Q+1+g_{x ; y} \\
& \psi_{6: x, y}=-f_{x, y}-g_{x ; y}
\end{aligned}
$$

For brevity, the multipliers $u_{i}=0$ if the coordinates $x-1$ or $y-1$ are outside their ranges in $\mathcal{R}$. Let $\xi_{i: x, y} \geq 0$ be nonnegative variables such that $u_{i: x, y} \xi_{i: x, y}=0$. Then the Karush-Kuhn-Tucker conditions for the QP problem of Eqs. (6) and (7) have the following form assuming that $(x, y) \in \mathcal{R}$, $(x-1, y) \in \mathcal{R}$, and $(x, y-1) \in \mathcal{R}$, respectively:

$$
\begin{aligned}
& -f_{x, y}+f_{x-1,1}-\xi_{1: x, y}=-\bar{\Delta}_{\mathrm{h}: x, y}+\delta g_{\mathrm{h}: x, y} \\
& f_{x, y}-f_{x-1, y}-\xi_{2: x, y}=\underline{\Delta}_{\mathrm{h}: x, y}-\delta g_{\mathrm{h}: x, y} \\
& -f_{x, y}+f_{x, y-1}-\xi_{3: x, y}=-\bar{\Delta}_{\mathrm{v}: x, y}+\delta g_{\mathrm{v}: x, y} \\
& f_{x, y}-f_{x, y-1}-\xi_{4: x, y}=\Delta_{\mathrm{v}: x, y}-\delta g_{\mathrm{v}: x ; y} \\
& -f_{x, y}-\xi_{5: x, y}=-Q+1+g_{x ; y} \\
& f_{x, y}-\xi_{6: x, y}=-g_{x ; y}
\end{aligned}
$$


and

$$
\begin{aligned}
2 f_{x, y} & +u_{1: x, y}-u_{2: x, y}-u_{1: x+1, y}+u_{2: x+1, y} \\
& +u_{3: x ; y}-u_{4: x, y}-u_{3: x, y+1}+u_{4: x, y+1} \\
& +u_{5 ; x, y}-u_{6 ; x, y}=0
\end{aligned}
$$

so that for all $(x, y) \in \mathcal{R}$

$$
\begin{aligned}
f_{x ; y}= & -\left(u_{1: x, y}-u_{2: x, y}-u_{1: x+1, y}+u_{2: x+1, y}\right. \\
& +u_{3: x ; y}-u_{4: x, y}-u_{3: x, y+1}+u_{4: x, y+1} \\
& \left.+u_{5 ; x, y}-u_{6 ; x, y}\right) / 2
\end{aligned}
$$

where $u_{i: x, y}=0$ if $i=1,2$ and $x=0$ and $i=3,4$ and $y=0$. The conditions of Eq. (8) lead to the the dual QP problem [14]:

$$
\min \left\{\boldsymbol{\Delta}^{\top} \mathbf{u}+\mathbf{u}^{\top} G \mathbf{u} \quad \mid \mathbf{u} \geq \mathbf{0}\right\}
$$

where the vectors $\boldsymbol{\Delta}$ represent the constraints of Eq. (3) and $\mathbf{u}$ the corresponding Lagrange multipliers and $G$ is the sparse matrix such that the equalities $2 G \mathbf{u}-\boldsymbol{\xi}=-\boldsymbol{\Delta}$ obtained by substituting Eq. (10) into Eq. (8) have the following form for all $(x, y) \in \mathcal{R}$ (due to the limited space, only 3 out of the 6 equations are shown below):

$$
\begin{aligned}
& u_{1: x, y}-u_{2: x, y}+\frac{1}{2}\left(-u_{1: x+1, y}+u_{2: x+1, y}\right. \\
& -u_{1: x-1, y}+u_{2: x-1, y}+u_{3: x, y}-u_{4: x, y} \\
& -u_{3: x, y+1}+u_{4: x, y+1}-u_{3: x-1, y}+u_{4: x-1, y} \\
& +u_{3: x-1, y+1}-u_{4: x-1, y+1} \\
& \left.+u_{5: x, y}-u_{6: x, y}-u_{5: x-1, y}+u_{6: x-1, y}\right) \\
& -\xi_{1: x, y}=-\bar{\Delta}_{\mathrm{h}: x, y}+\delta g_{\mathrm{h}: x, y} \\
& \hline-u_{1: x, y}+u_{2: x, y}+\frac{1}{2}\left(u_{1: x+1, y}-u_{2: x+1, y}\right. \\
& +u_{1: x-1, y}-u_{2: x-1, y}-u_{3: x, y}+u_{4: x, y} \\
& +u_{3: x, y+1}-u_{4: x, y+1}+u_{3: x-1, y}-u_{4: x-1, y} \\
& -u_{3: x-1, y+1}+u_{4: x-1, y+1} \\
& \left.-u_{5: x ; y}+u_{6: x ; y}+u_{5: x-1, y}-u_{6: x-1, y}\right) \\
& -\xi_{2: x, y}=\underline{\mathrm{h}}_{\mathrm{h}: x, y}-\delta g_{\mathrm{h}: x, y} \\
& \ldots \\
& \quad \ldots \\
& \frac{1}{2}\left(-u_{1: x, y}+u_{2: x, y}+u_{1: x+1, y}-u_{2: x+1, y}\right. \\
& -u_{3: x, y}+u_{4: x, y}+u_{3: x, y+1}-u_{4: x, y+1} \\
& \left.-u_{5: x, y}+u_{6: x, y}\right)-\xi_{6: x, y}=-g_{x, y}
\end{aligned}
$$

The linear system of inequalities (11)-(13) under the conditions $\boldsymbol{\xi} \geq \mathbf{0} ; \mathbf{u} \geq \mathbf{0}$, and $\mathbf{u}^{\top} \boldsymbol{\xi}=0$ is solved by an iterative process similar to the GaussSeidel coordinate relaxation process for systems of linear equations $[12,13,14]$. For the lattice $\mathbf{R}$ with $M N$ pixels, the vector $\mathbf{u}$ has $(M-1) N$ components $u_{1: x, y}$ and $(M-1) N$ components $u_{2: x, y}$ for $x=1, \ldots, M-1$ and $y=0, \ldots, N-1 ; M(N-$ 1) components $u_{3: x, y}$ and $M(N-1)$ components $u_{4: x, y}$ for $x=0, \ldots, M-1$ and $y=1, \ldots, N-1$, and $M N$ components $u_{5: x, y}$ and $M N$ components $u_{6: x, y}$ for $x=0, \ldots, M-1$ and $y=0, \ldots, N-$ 1. An individual iteration replaces each successive component with a new value, $u_{\ldots}=\max \left\{0, w_{\ldots}\right\}$, computed using the fixed current values of all other components, e.g. $w_{1: ; x, y}=\left(-\bar{\Delta}_{\mathrm{h}: x, y}+\delta g_{\mathrm{h}: x ; y}\right)+u_{2: x, y}$

$-\frac{1}{2}\left(-u_{1: x+1, y}+[\right.$ other terms from Eq. (11)])

$w_{2: x, y}=\left(\underline{\Delta}_{\mathrm{h}: x, y}-\delta g_{\mathrm{h}: x, y}\right)+u_{1: x, y}$

$-\frac{1}{2}\left(u_{1: x+1, y}-[\right.$ other terms from Eq. (12)])

...

$w_{6: x ; y}=-2 g_{x, y}+u_{5: x, y}$

- [other terms from Eq. (13)]

After this process converges to the final $u$-values, the transformed template $a$ that provides the minimum matching score $d(g, t \mid E)$ of Eq. (5) is obtained using Eq. (10): $a_{x, y}=f_{x, y}+g_{x, y}$.

In geometric terms, the solution of the dual QP problem starts outside the constrained domain of Eq. (8) and converges to the solution (Eq. (5)).

\begin{tabular}{|c|c|c|c|c|c|}
\hline$\alpha$ & 0 & 45 & & & 75 \\
\hline$\beta$ & $\begin{array}{llll}15 & 45 & 75 & 90\end{array}$ & 15 & $45 \quad 75$ & 90 & $\begin{array}{llll}15 & 45 & 75 & 90\end{array}$ \\
\hline $\mathrm{C}_{1}$ & $\begin{array}{llll}0 & 7.8 & 12 & 14\end{array}$ & $\overline{12}$ & 1112 & 13 & $\begin{array}{llll}12 & 12 & 12 & 12\end{array}$ \\
\hline $\mathrm{C}_{2}$ & $\begin{array}{llll}0 & 7.8 & 12 & 13\end{array}$ & 10 & $11 \quad 12$ & 12 & $\begin{array}{llll}12 & 12 & 11 & 11\end{array}$ \\
\hline $\mathrm{C}_{3}$ & $\begin{array}{llll}0 & 7.1 & 10 & 12\end{array}$ & 10 & $11 \quad 12$ & 12 & $\begin{array}{llll}12 & 12 & 11 & 11\end{array}$ \\
\hline $\mathrm{C}_{4}$ & $\begin{array}{llll}0 & 6.5 & 10 & 11\end{array}$ & 10 & $10 \quad 11$ & 11 & $\begin{array}{llll}12 & 11 & 11 & 11\end{array}$ \\
\hline $\bar{Q}$ & $\begin{array}{lllll} & 1.5 & 3.2 & 4.7\end{array}$ & 2.8 & 2.94 .1 & 4.7 & 4.74 .74 .84 .9 \\
\hline
\end{tabular}
Convergence is reached when the maximum violations of the constraints

$$
\begin{aligned}
\varepsilon_{1} & =\max _{x, y}\left\{\delta a_{\mathrm{h}: x, y}-\bar{\Delta}_{\mathrm{h}: x, y}, 0\right\} \\
\varepsilon_{2} & =\max _{x, y}\left\{-\delta a_{\mathrm{h}: x, y}+\underline{\Delta}_{\mathrm{h}: x, y}, 0\right\} \\
\varepsilon_{3} & =\max _{x, y}\left\{\delta a_{\mathrm{v}: x, y}-\bar{\Delta}_{\mathrm{v}: x, y}, 0\right\} \\
\varepsilon_{4} & =\max _{x, y}\left\{-\delta a_{\mathrm{v}: x, y}+\underline{\Delta}_{\mathrm{v}: x, y}, 0\right\} \\
\varepsilon_{5} & =\max _{x, y}\left\{a_{x, y}-Q+1,0\right\} \\
\varepsilon_{6} & =\max _{x, y}\left\{-a_{x, y}, 0\right\}
\end{aligned}
$$

are below a fixed threshold.

\section{Experiments and Conclusions}

Our experiments used the MIT face database [8] containing 36 face images for each of ten persons. Illumination directions vary from $0^{\circ}$ to $75^{\circ}$ vertically and from $15^{\circ}$ to $90^{\circ}$ horizontally with increments of $15^{\circ}$. The image with almost frontal illumination $\left(0^{\circ}, 15^{\circ}\right)$ was selected as the prototype, $t$, for matching to other images. Figure 5 shows a subset of images of the same face from this database.

Table 1: Average absolute pixel-wise squared distance, $\left.s=\sqrt{(} \frac{1}{X Y} D(g, t \mid E)\right)$ between the image $g$ and the closest transformed template, $a$, for 36 faces "MIT00004" from the MIT database ( $\alpha$ and $\beta$ - the vertical and horizontal illumination angles, respectively); $\mathrm{C}_{i}-$ concurrent correlation matching with $i^{\text {th }}$ order polynomial model, and Q-QP-based matching.

Transformations of the template by concurrent correlation matching to the other images using the first and second order polynomial contrast and offset models are shown in Figs. 6 and 7, respectively. 
0
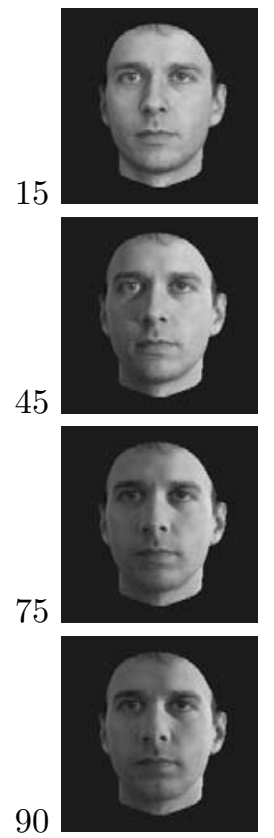

45
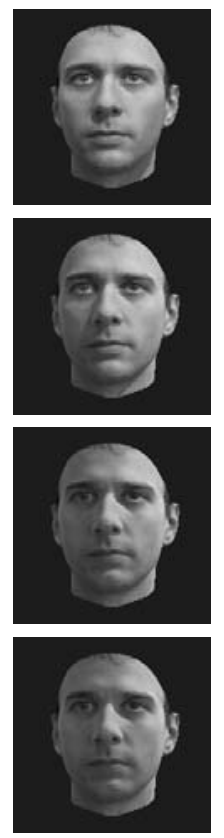

75
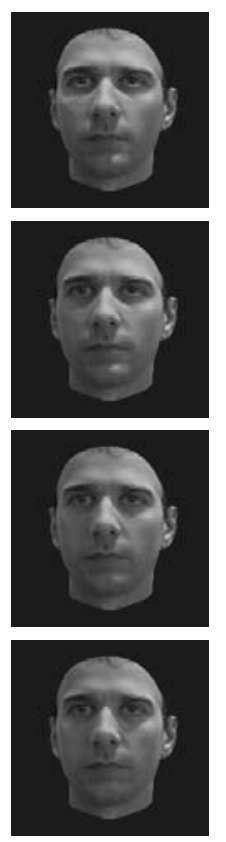

Figure 5: Face "MIT-00004" [8] with varying vertical $\left(0^{\circ}-75^{\circ}\right)$ and horizontal $\left(15^{\circ}-90^{\circ}\right)$ illumination directions.

0
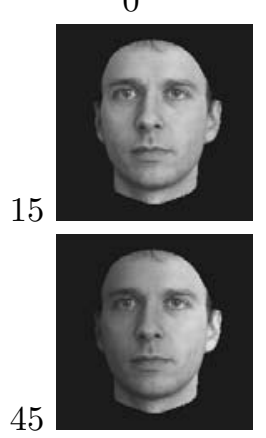

45
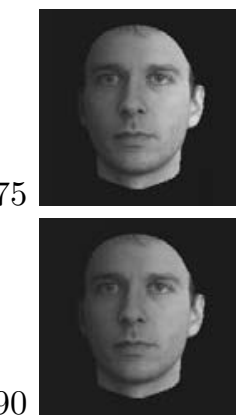

45
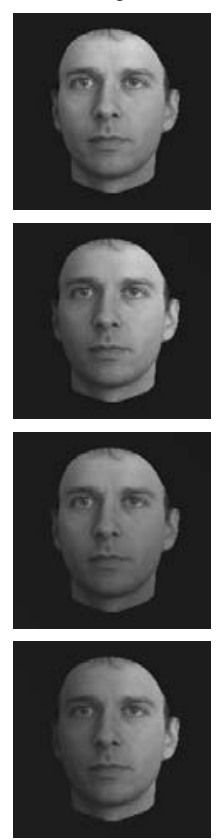

75
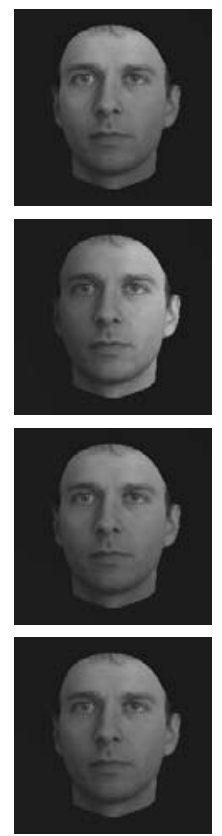

Figure 6: Concurrent correlation: template $\left(0^{\circ} ; 15^{\circ}\right)$ transformed under the first order polynomial model to match the images in Fig. 5.

Results for QP-matching are given in Fig. 8. Table 1 shows the average residual absolute distances between the matched image, $g$, and the closest transformed template, $a$, in Eq. (5). The QP iterations were terminated after the maximum violation of the constraints in Eq. (17) became less than 1 from 85 to 780 iterations in Fig. 8. These and similar experiments with other images from the same

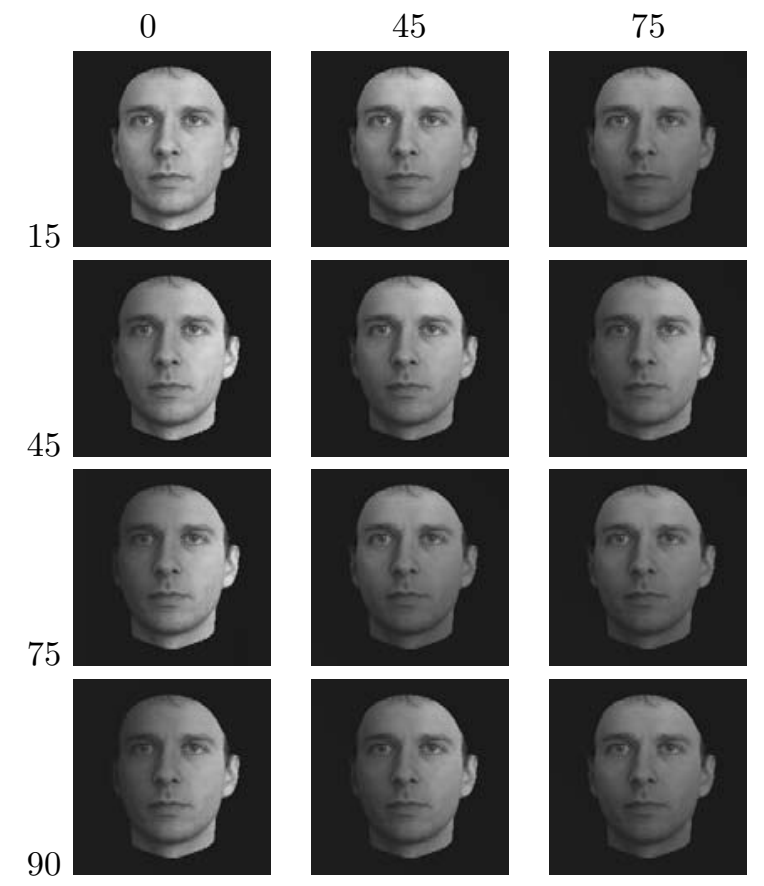

Figure 7: Concurrent correlation: template $\left(0^{\circ} ; 15^{\circ}\right)$ transformed under the second order polynomial model to match images in Fig. 5.

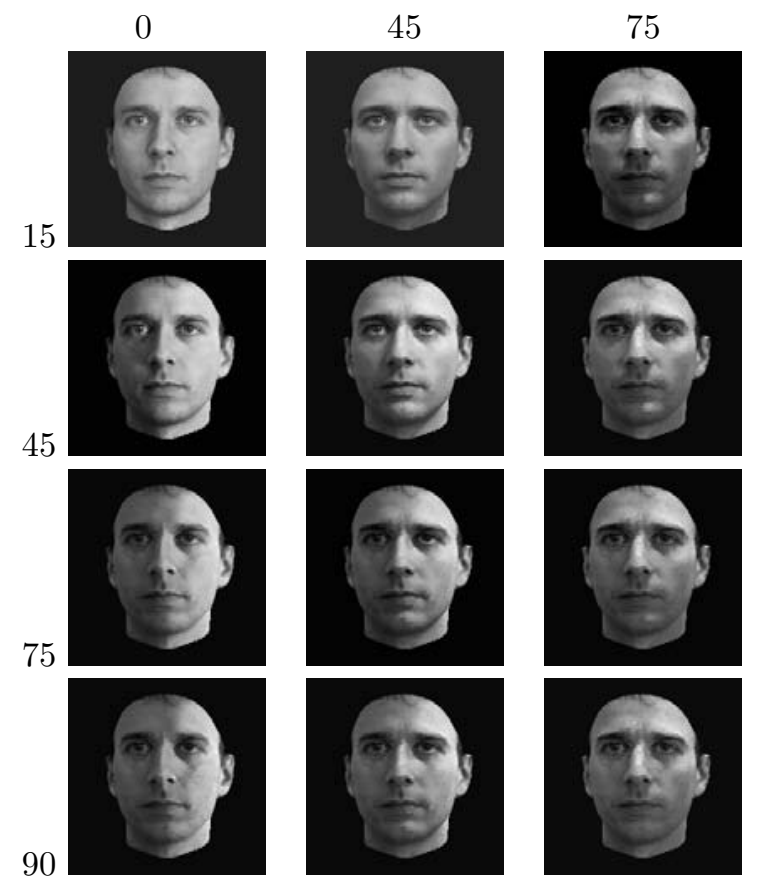

Figure 8: QP matching: template $\left(0^{\circ} ; 15^{\circ}\right)$ transformed in the local range $E=[0.25 . .4 .0]$ to match images in Fig. 5.

database showed that the proposed general model and QP matching allow us to account better for natural non-uniform contrast and offset deviations due to varying illumination than up to $4^{\text {th }}$ order polynomial models.

Table 2 compares QP-matching to Lai's matching algorithm with the quadratic polynomial contrast 
model on the MIT database [8] by showing the mean $\left(D_{\mathrm{mn}}\right)$, standard deviation $\left(\sigma_{D}\right)$, minimum $\left(D_{\min }\right)$, and maximum $\left(D_{\max }\right)$ values of the matching scores $D(g, t \mid E)$ in Eq. (5). These experiments involved 30 template-target image pairs such that different images of each person are matched only to their own template $\left(0^{\circ}, 15^{\circ}\right)$ and the test targets $g$ are either synthetic (namely, the template $t$ transformed by arbitrary uniform contrast and offset changes or quadratic contrast variations shown in Fig. 1(b)), or natural MIT database images with large illumination differences with respect to the templates.

Table 2: Distributions of the matching scores for the QP-matching and Lai's approach (within the brackets) under synthetic uniform (SU); synthetic quadratic $(\mathrm{SQ})$, and (NNU) natural non-uniform contrast and offset deviations.

\begin{tabular}{rcccc} 
Test & $D_{\text {mn }}$ & $\sigma_{D}$ & $D_{\min }$ & $D_{\max }$ \\
\hline SU & $\mathbf{1 . 7}[23]$ & $\mathbf{0 . 4}[3.7]$ & $\mathbf{0 . 4}[15]$ & $\mathbf{7 . 1}[29]$ \\
SQ & $64[\mathbf{6 . 1}]$ & $1.6[\mathbf{7 . 6}]$ & $49[\mathbf{0 . 8}]$ & $85[\mathbf{2 8}]$ \\
NNU & $\mathbf{4 . 4}[62]$ & $\mathbf{0 . 6}[39]$ & $\mathbf{0 . 2}[39]$ & $\mathbf{1 1}[150]$ \\
\hline
\end{tabular}

Excepting the global quadratic contrast variations, our QP-based matching for the general non-uniform contrast and offset model outperformed Lai's approach based on the polynomial model. The latter matching works better in these experiments for the quadratic contrast changes only because our general model (Eq. (3)) forbids sign changes in local signal differences in a template to preserve better the natural appearance of the transformed images whereas the quadratic contrast factors allow sign changes. Due to relatively simple repetitive computations at each step, QP-based matching offers good prospects of specialised hardware implementation.

\section{References}

[1] P. Aschwanden and W. Guggenbühl, "Experimental results from a comparative study on correlation-type registration algorithms," in Robust Computer Vision, W. Förstner and S. Ruwiedel, Eds. Karlsruhe, Germany: Wichmann, 1992, pp. 268-282.

[2] J. Crowley and J. Martin, "Experimental comparison of correlation techniques," in IAS-4, International Conference on Intelligent Autonomous Systems, Karlsruhe, March, 1995.

[3] S. Lai, "Robust image matching under partial occlusion and spatially varying illumination change," Computer Vision and Image Understanding, vol. 78, no. 1, pp. 84-98, 2000.

[4] J. Chen, C. Chen, and Y. Chen, "Fast algorithm for robust template matching with M-estimators," IEEE Trans. Signal Processing, vol. 51, no. 1, pp. 230-243, 2003.
[5] C. Yang, S. Lai, and L. Chang, "Robust face image matching under illumination variations," EURASIP Journal on Applied Signal Processing, vol. 2004, no. 16, pp. 2533-2543, 2004.

[6] S. Wei and S. Lai, "Robust and efficient image alignment based on relative gradient matching," Image Processing, IEEE Transactions on, vol. 15, no. 10, pp. 2936-2943, 2006.

[7] P. Huber, Robust Statistics. N.Y.: Wiley, 1981.

[8] B. Weyrauch, J. Huang, B. Heisele, and V. Blanz, "Component-based face recognition with $3 \mathrm{~d}$ morphable models," in CD-ROM Proc. First IEEE Workshop on Face Processing in Video, Washington D.C., June 28, 2004, 2004.

[9] P. Delmas, G. L. Gimel'farb, A. Shorin, and J. Morris, "Robust least-squares image matching in the presence of outliers," in Proc. 12 ${ }^{\text {th }}$ Int. Conf. Computer Analysis of Images and Patterns (CAIP 2007), Vienna, Austria, August 2007, ser. Lecture Notes in Computer Science, vol. 4673. Berlin: Springer, 2007, pp. 776-783.

[10] G. Gimel'farb, P. Delmas, J. Morris, and A. Shorin, "Robust face matching under large occlusions," in Proc. $14^{\text {th }}$ Int. Conf. Image Analysis and Processing (ICIAP 2007), Modena, Italy, Sept. 200\%. Los Alamitos: IEEE Computer Society Press, 2007, pp. 448-453.

[11] M. Turk and A. Pentland, "Face recognition using eigenfaces," in Proc. IEEE Computer Soc. Conf. Computer Vision and Pattern Recognition (CVPR'91), Lahaiana, Maui, Hawaii, June 3-6, 1991. Los Alamitos: IEEE Computer Society Press, 1991, pp. 586-591.

[12] C. Hildreth, "A quadratic programming procedure," Naval Research Logistics Quarterly, vol. 4, pp. 79-85, 1957.

[13] D. D'Esopo, "A convex programming procedure," Naval Research Logistics Quarterly, vol. 6, pp. 3342, 1959.

[14] H. Künzi and W. Krelle, Nichtlineare programmierung. Berlin: Springer, 1962. 